\title{
Sintomas depressivos e aspectos subjetivos associados à cefaleia do tipo primária
}

\author{
Amanda Santos de Souza \\ Kelly Larissa de Souza Miranda \\ Roberta Ferrari Marback
}

\section{RESUMO}

A cefaleia é um sintoma muito comum na rotina das pessoas e uma queixa frequente na prática dos profissionais de saúde. Caracteriza-se como primária e secundária, sendo a primária aquela que não possui etiologia que a explique a partir de exames clínicos ou laboratoriais. Estudos têm apontado a frequente associação entre cefaleia primária e depressão, bem como mostrado os efeitos trazidos pela cefaleia à vida das pessoas acometidas. Desse modo, o presente estudo teve como objetivo geral identificar a relação entre sintomas depressivos, aspectos subjetivos e cefaleia primária. Trata-se de um estudo transversal no qual foram aplicados questionário sociodemográfico, entrevista semiestruturada, Escala Visual Analógica (EVA) e Inventário de Depressão de Beck (BDI) em 40 participantes de ambos os sexos, maiores de 18 anos, que apresentavam cefaleia primária. Todos os participantes autorizaram sua participação na pesquisa mediante assinatura do Termo de Consentimento Livre e Esclarecido. Constatou-se que 52,5\% dos participantes apresentaram sintomas depressivos nos níveis leve a grave, sendo os sentimentos de tristeza e angústia os mais comuns perante a dor.

Palavras-chave: cefaleia; cefaleia primária; sintomas depressivos; depressão; aspectos subjetivos.

\section{ABSTRACT}

\section{Depressive symptoms and subjective aspects associated with primary headache}

Headache is a very common symptom in the people's routine and a frequent complaint in the practice of health professionals. It is characterized as primary and secondary. The primary one is the one that cannot be explained by clinical or laboratory tests. Studies show a frequent association between primary headache and depression as well as it shows the effects brought about by headache to the lives of affected people. Therefore, the aim of the present study is to identify the relevance between depressive symptoms, subjective aspects and primary headache. This was a cross-sectional study in which the sociodemographic questionnaire, semi-structured interview, Visual Analogue Scale (VAS) and Beck Depression Inventory (BDI) were applied to 40 people of mixed-gender group, over 18 years old, who were having primary headache. All the participants authorized their participation in the research by signing the Informed Consent Term. It was found that $52.5 \%$ of the people were having depressive symptoms from mild to severe levels, being sadness and anguish the most common feelings in face of pain.

Keywords: headache; primary headache; depressive symptoms; depression; subjective aspects.

A dor crônica é uma condição alarmante do ponto de vista da saúde pública, já que interfere significativamente no funcionamento, produção, relações sociais e, principalmente, na qualidade de vida das pessoas acometidas (Lima \& Trad, 2007). Dentre os tipos de dor

\section{Sobre os Autores}

A.S. S.

orcid.org/0000-0002-9874-2541

Universidade Salvador

(UNIFACS) - Salvador, BA

amandasouza.s@hotmail.com

K. L. S. M.

orcid.org/0000-0001-7996-5531

Universidade Salvador

(UNIFACS) - Salvador, BA

kellymiranda.fdj@gmail.com

R. F. M.

orcid.org/0000-0003-3870-8963

Universidade Salvador

(UNIFACS) - Salvador, BA

roberta.marback@pro.unifacs.b $r$

\section{Direitos Autorais}

Este é um artigo de acesso aberto e pode ser reproduzido livremente, distribuído,

transmitido ou modificado, por qualquer pessoa desde que usado sem fins comerciais. 0 trabalho é disponibilizado sob a licença Creative Commons CCBY-NC.

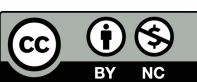




\section{W'INTERACÃO EM ET PSICOLOGIA}

crônica, as cefaleias se destacam por apresentarem alta prevalência e por se tratar de uma condição incapacitante, aparecendo como a segunda queixa mais comum de dor (Organização Mundial da Saúde - OMS, 2001). Estima-se que mais da metade da população apresente cefaleia, inclusive de forma crônica, em algum momento da vida (Nobre, 2006), representando cerca de $9 \%$ das consultas em atenção primária (Subcomitê de Classificação das Cefaleias da Sociedade Internacional das Cefaleias, 2006) e gerando altos custos ao sistema de saúde (Pinto et al., 2007).

As cefaleias se classificam em primárias e secundárias, de acordo com a etiologia. Diferentemente da secundária, que ocorre como resultado de outra patologia esclarecida, a cefaleia primária não é explicada por meio de exames laboratoriais ou clínicos, sendo as mais comuns a do tipo tensional, caracterizada por dores que provocam sensação de cabeça pesada/pressionada, de intensidade fraca ou moderada, sem impedimento de realização das atividades diárias; e migrânea, também denominada enxaqueca, que incorpora a manifestação de crises repetidas cuja dor envolve parte da cabeça e se associa a outros sintomas como náuseas, vômitos, fotofobia e sensibilidade aos sons (Speciali, 1997; Krymchantowski, 2008). De acordo com Tsuji e Carvalho (2002), pessoas acometidas por crises recorrentes desse tipo de cefaleia relatam sentir dores fortes, marcadas principalmente por muito incômodo e angústia, devido ao desconhecimento dos fatores patológicos que causam os sintomas.

Tal condição compromete a qualidade de vida dessas pessoas de maneira sistêmica, muitas vezes implicando o desenvolvimento de comorbidades psiquiátricas, dentre elas a depressão, a qual possui uma prevalência de $17 \%$ ao longo da vida e cuja sintomatologia inclui aspectos orgânicos, comportamentais e psicológicos bastante característicos, dentre eles: tristeza frequente, avolia, rebaixamento do humor, distúrbios do sono, diminuição do apetite e/ou libido. Do ponto de vista etiológico, pode-se dizer que a depressão é resultado de um conjunto de fatores (ambientais, psicológicos, genéticos, orgânicos etc.) que parece estar associado a uma disfunção de neurotransmissores, principalmente dopamina, noradrenalina e serotonina (American Psychiatric Association, 2014).

Freud (1893) sugeriu a correlação entre a dor física e a psíquica, afirmando que a melancolia poderia se manifestar por meio de somatizações. Uma vez que a dor consiste em uma manifestação sintomática da depressão, e tendo em vista que tal quadro psicopatológico se caracteriza pela exacerbação de sensações desagradáveis, torna-se mais clara a compreensão da associação de ambas as condições.

Ainda nesse sentido, Petersen e Nunes (2002) constataram que há uma associação entre cefaleia e depressão manifesta, visto que $50 \%$ das pessoas acometidas pela cefaleia apresentaram sintomas depressivos e $17 \%$ apresentaram escores abaixo do normal para o Inventário de Depressão de Beck, utilizado no referido estudo, apontando assim um provável movimento de negação do quadro depressivo. Nesse estudo, a incidência de depressão foi aproximadamente cinco vezes mais alta do que na população geral. Ainda, Feldman (1995) constatou em seu estudo que $46 \%$ dos participantes com cefaleias apresentaram depressão.

De acordo com Breslau, Davis, Schultz, e Peterson (1994), existem diversas similaridades clínicas e epidemiológicas entre enxaqueca e depressão maior: ambas ocorrem principalmente em adultos jovens, têm prevalência equivalente na população geral e acometem mais pessoas do sexo feminino ( $24 \%$ em mulheres e $9 \%$ em homens, no caso da migrânea; $24 \%$ em mulheres e $13 \%$ em homens, na depressão maior). Além disso, nota-se que no momento dos ataques de enxaqueca, algumas pessoas apresentam mudanças no humor e/ou no comportamento.

A literatura não comporta produções suficientes acerca da temática, embora exista uma relação entre cefaleia e depressão e variáveis psicológicas contribuam para a manifestação e/ou intensificação dos episódios de cefaleia; segundo Costa, Ybarra, Côrrea, e Teixeira (2006) a depressão atinge três vezes mais a população com migrânea do que a população geral. Partindo da compreensão de que a comorbidade psiquiátrica complica o manejo da cefaleia por piorar a resposta ao tratamento e dificultar o prognóstico, torna-se fundamental compreender de forma mais clara a relação entre cefaleia e depressão, no intuito de otimizar o tratamento e o suporte psicológico oferecidos aos indivíduos acometidos.

Desse modo, o presente estudo propôs-se a investigar a incidência de sintomas depressivos e aspectos subjetivos associados à cefaleia primária, mediante a compreensão da repercussão, interferência e autopercepção do sujeito acometido em relação à cefaleia.

\section{MÉTODO}

Estudo transversal, quantitativo e qualitativo, realizado com 40 indivíduos de ambos os sexos, maiores de 18 anos. A amostra foi selecionada por demanda espontânea e acessibilidade, sendo composta por pessoas que apresentavam cefaleia primária. As pesquisadoras fizeram busca ativa dos participantes, realizando encontros em locais favoráveis à coleta de dados, os quais ocorreram predominantemente na clínica escola de Psicologia da 
Universidade Salvador (UNIFACS). Todos os participantes incluídos no estudo informaram ter passado por uma bateria de exames médicos que descartavam a possibilidade de uma explicação etiológica para a manifestação da dor de cabeça, bem como já haviam submetido-se a, pelo menos, um tratamento para a cefaleia. Aqueles que relataram sentir dor de cabeça devido a problemas secundários, de ordem neurológica, oftalmológica, dentre outras, foram excluídos do estudo.

Para a coleta dos dados, foram utilizados os seguintes instrumentos: entrevista semiestruturada, elaborada pelas pesquisadoras, para investigação das características e dos aspectos subjetivos associados à cefaleia; questionário sociodemográfico para caracterização dos participantes em relação à idade, escolaridade, ocupação, renda familiar, estado civil e religião; Escala Visual Analógica (EVA), instrumento de avaliação da intensidade da dor utilizado em diversos contextos clínicos, que, para o presente estudo, serviu de parâmetro avaliativo da intensidade da cefaleia. Trata-se de uma régua colorida e numerada de 0 a 10, sendo 0 a numeração correspondente à ausência total de dor e 10 o nível de dor máxima suportável pelo sujeito. A escala subdivide-se em 3 categorias: leve, que engloba os números de 0 a 2; moderada, que corresponde a numeração de 3 a 7; e intensa, que vai de 8 a 10. É importante salientar que as cores da escala tinham como função enfatizar o aumento da dor em ordem crescente, iniciando com 0 , em tonalidade azul claro, e finalizando com vermelho intenso, no número 10 (Rubbo, 2010).

Por fim, foi aplicado o Inventário de Depressão de Beck (BDI), instrumento de autorrelato em formato de questionário de múltipla escolha, que tem por objetivo mensurar sintomas depressivos como desesperança, irritabilidade, sentimentos de culpa, punição, dentre outros. Composto por 21 categorias de sintomas e atitudes, os resultados do instrumento são avaliados com base na soma do escore total, visto que cada item contempla pontuações que variam de 0 a 3 . 0 Escores iguais ou inferiores a 9 não indicam depressão, ao passo que somas variadas entre 10-18 indicam depressão leve a moderada, 19-29 depressão moderada a severa, e 30-63 depressão severa (Beck, Ward, Mendelson, Mock, \& Erbaugh, 1961).

A análise de dados foi realizada quantitativa e qualitativamente, de acordo com a demanda de cada instrumento. As entrevistas foram analisadas em conjunto com o questionário sociodemográfico, sendo submetidas à análise de conteúdo. Essa técnica é utilizada para interpretar e descrever o conteúdo de um conjunto de textos e/ou documentos, com o objetivo de alcançar uma compreensão mais ampla de seus significados (Bardin, 1977).
No que concerne à avaliação dos aspectos subjetivos, foram coletados e analisados os dados acerca de sentimentos, pensamentos e comportamentos oriundos da manifestação da cefaleia, bem como a representação simbólica dessa condição na vida dos acometidos. $\mathrm{Na}$ maioria dos casos, os participantes referiram mais de uma resposta para cada categoria, sendo todas computadas e, algumas, agregadas em um único grupo. 0 critério utilizado para tal agregação foi a similaridade das respostas.

A análise da EVA se deu por meio do cálculo da média aritmética dos resultados encontrados após sua aplicação, enquanto o BDI foi analisado com base nas instruções do manual, a partir da construção de tabelas acerca da sintomatologia da depressão.

Todos os participantes autorizaram sua participação na pesquisa, mediante assinatura do Termo de Consentimento Livre e Esclarecido, tendo recebido explicações prévias acerca do estudo, da importância da colaboração e da gravação da entrevista para melhor aproveitamento dos dados. Além disso, foram assegurados sigilo e anonimato das informações, bem como dos princípios éticos determinados pela Resolução 466/2012 (Brasil, 2012).

A pesquisa foi previamente aprovada pelo Comitê de Ética e Pesquisa do setor de saúde da Universidade Salvador (UNIFACS), CAAE $n^{\circ}$ 47303115.1.0000.5033, tendo sido desenvolvida entre fevereiro de 2016 e fevereiro de 2017, no programa de Iniciação Científica do curso de Psicologia da Universidade Salvador (UNIFACS).

\section{RESULTADOS}

Participaram do estudo 40 indivíduos, sendo $87,5 \%$ do sexo feminino e $12,5 \%$ do sexo masculino, com média de idade de 32,5 anos. No que se refere ao estado civil, $60 \%$ eram solteiros, $30 \%$, casados e $10 \%$, divorciados. Quanto à escolaridade, $50 \%$ dos participantes possuíam ensino superior incompleto, $30 \%$ possuíam ensino superior completo, $10 \%$ possuíam ensino médio completo e $10 \%$ não possuíam ensino médio completo. No que se refere à religião, a maioria dos participantes $(42,5 \%)$ era católica; $22 \%$, evangélicos; e $20 \%$ não possuíam religião. Quanto à ocupação, 35\% apenas estudavam e 12,5\% trabalhavam com comércio. A renda familiar de $35 \%$ dos participantes estava acima de 7 salários mínimos; $20 \%$, entre 5 e 7 salários mínimos; $15 \%$, entre 3 e 5 salários mínimos; $15 \%$, entre 2 e 3 salários mínimos; e 15\%, até um salário mínimo.

Em relação à manifestação do primeiro episódio de cefaleia encontrou-se uma média de 17,5 anos; $55 \%$ dos participantes informaram que não houve motivo aparente para a primeira manifestação de dor; $20 \%$ apontaram como 


\section{W'INTERACÃO EM ET PSICOLOGIA}

motivo fatores externos (estresse e importantes mudanças na vida pessoal); e $15 \%$ associaram tal manifestação à menarca e questões hormonais.

Como desencadeador, $47,5 \%$ dos participantes afirmaram que fatores emocionais como estresse, ansiedade e cansaço costumavam ser os principais responsáveis pelo aparecimento da cefaleia; $30 \%$ apontaram fatores físicos (questões hormonais e menstruação); $25 \%$ relataram que a má alimentação, ingestão de alimentos específicos e/ou a fome desencadeiam a dor de cabeça; e 12,5\% dos participantes atribuíram como desencadeador do episódio de cefaleia a má qualidade do sono e/ou sua privação.

Com relação à frequência dos episódios de cefaleia, 25\% afirmaram sentir dor de cabeça todos ou quase todos os dias; $52,5 \%$ afirmaram sentir de 1 a 4 vezes na semana; e 7,5\% afirmaram sentir apenas em momentos de estresse e/ou ansiedade. No que diz respeito à intensidade da dor, a análise da aplicação da EVA apontou que a maioria dos participantes sofre de dores medianas a intensas, sendo a média geral do instrumento igual a 7,1 . No que se refere às respostas do BDI, constatou-se que $47,5 \%$ dos participantes apresentaram nível mínimo de depressão; 20\% apresentaram nível leve; 30\% nível moderado; e 2,5\% nível grave.

Com relação aos aspectos subjetivos, sua análise mostrou quais os sentimentos/emoções, pensamentos e comportamentos mais referidos pelos participantes nos momentos em que sentiam dor de cabeça, além da representação simbólica dos quadros sintomáticos. No que cerne aos sentimentos/emoções, aqueles citados mais frequentemente foram: tristeza, angústia, impaciência, irritação, mau humor e estresse. É importante ressaltar que a maioria dos participantes referiu mais de um sentimento/ emoção, e todas as respostas foram computadas, sendo apontadas as mais frequentes.

Os pensamentos, de modo geral, estavam relacionados à busca pela solução e livramento da dor, sendo a utilização de medicamentos a principal estratégia utilizada. Quatro participantes referiram pensamentos suicidas. Em termos de comportamento, os mais comuns incluíram tomar banho, usar medicamentos analgésicos, isolar-se e relaxar por meio de alguma atividade prazerosa ou que pudesse distrair.

Com relação ao significado da dor de cabeça, ou seja, sua representação simbólica, os participantes relataram que se tratava de algo ruim, desconfortável e incapacitante. Finalmente, apontaram que, nos momentos de dor, as pessoas com quem se relacionavam no cotidiano se mostravam mais solidárias e cuidadosas. A Tabela 1 ilustra os aspectos subjetivos (sentimentos, pensamentos, comportamentos e representações simbólicas) informados pelos respondentes de modo mais prevalente.

\begin{tabular}{|c|c|c|}
\hline \multicolumn{2}{|c|}{ Aspectos subjetivos } & $\begin{array}{l}\text { Número de } \\
\text { respostas }\end{array}$ \\
\hline \multirow{4}{*}{$\begin{array}{l}\text { Emoção/ } \\
\text { sentimento }\end{array}$} & Angústia e tristeza & 13 \\
\hline & Impaciência e irritação & 9 \\
\hline & Estresse & 6 \\
\hline & Ansiedade & 4 \\
\hline \multirow{4}{*}{ Pensamentos } & $\begin{array}{l}\text { "Preciso solucionar } \\
\text { essa situação" }\end{array}$ & 15 \\
\hline & "Vou tomar remédio" & 7 \\
\hline & "Quero morrer" & 4 \\
\hline & "Preciso me distrair" & 2 \\
\hline \multirow{4}{*}{ Comportamentos } & Tomar remédio & 17 \\
\hline & Dormir/descansar & 15 \\
\hline & Isolar-se & 14 \\
\hline & Relaxar & 14 \\
\hline \multirow{4}{*}{$\begin{array}{l}\text { Representação } \\
\text { simbólica }\end{array}$} & $\begin{array}{l}\text { "É literalmente uma } \\
\text { dor de cabeça" }\end{array}$ & - \\
\hline & $\begin{array}{l}\text { "É um freio, me freia } \\
\text { de fazer alguma coisa" }\end{array}$ & - \\
\hline & "É uma maldição" & - \\
\hline & "É uma cruz" & - \\
\hline
\end{tabular}

Os sentimentos de angústia e tristeza no momento da dor foram referidos por $32,6 \%$ dos entrevistados, e $22,5 \%$ disseram sentir-se irritados e/ou impacientes. 0 estresse foi citado por $15 \%$, e $10 \%$ sentiam-se ansiosos. Quanto ao comportamento no momento da dor, $42,5 \%$ responderam que tomavam remédio; $37,5 \%$ procuravam dormir/descansar para tentar aliviar a dor; e $35 \%$ buscavam isolamento e atividades que proporcionassem relaxamento. Os pensamentos mais prevalentes giravam em torno da solução do problema e da procura de distrações. As representações simbólicas, de modo geral, evidenciaram o caráter incapacitante e a angústia provocados pelas crises de cefaleia, apontando seu aspecto limitador no que concerne à execução de atividades, bem como a associação do quadro sintomático a um fardo a ser encarado pelo sujeito acometido.

\section{DISCUSSÃO}

Os dados referentes ao sexo e nível de escolaridade encontrados no presente estudo corroboram informações observadas na literatura (Flores \& Costa, 2004). De acordo com esses autores, a cefaleia episódica ocorre mais predominantemente em pessoas do sexo feminino, com nível educacional mais elevado, dado compatível com o que foi encontrado na presente pesquisa, uma vez que a maioria dos participantes eram do sexo feminino $(87,5 \%)$, com nível 


\section{W'INTERACÃO EM ET PSICOLOGIA}

educacional relativamente elevado (apenas 4 participantes não tinham ensino médio completo), e cuja frequência dos episódios de cefaleia variaram de 1 a 4 vezes por semana para $52,5 \%$ dos participantes, enquanto $7,5 \%$ sentiam apenas em momentos de estresse e ansiedade.

Ainda de acordo com os autores, a faixa etária atingida por essa população costuma variar dos 20 aos 50 anos (Flores \& Costa, 2004), dado que também corrobora o que foi encontrado no presente estudo, haja vista a média de idade ter correspondido a 32,5 anos. Outro estudo cujos resultados são compatíveis indica que a média de idade das pessoas acometidas é de 34,9 anos (Magalhães, Lopes, \& Marback, 2016). Tais achados remetem-se à compreensão de que se trata de uma faixa etária na qual as pessoas encontram-se mais implicadas no âmbito laboral e acadêmico, e que representa o "auge" da vida ativa (Zukerman, Guendler, Mercante, \& Peres, 2004), o que pode sugerir uma relação entre engajamento profissional e trabalho com a manifestação sintomática de dor.

Ainda em relação à prevalência, vários estudos apontam que a cefaleia acomete mais pessoas do sexo feminino (Flores \& Costa, 2004; Morais \& Benseñor, 2009; Petersen \& Nunes, 2002; Villa, 2015), tornando possível hipotetizar que fatores hormonais possam estar relacionados à etiologia da doença, já que em muitos casos as crises de dor podem piorar no período menstrual e melhorar na menopausa e durante a gravidez (Bigal, Bordini, \& Speciali, 1999; Bordini, Bigal, \& Silva, 2001 como citado em Flores \& Costa, 2004). De modo compatível a tais achados, o presente estudo constatou que $30 \%$ dos entrevistados apontaram a menarca como desencadeador da primeira crise e a menstruação como intensificador das crises episódicas de cefaleia.

Acerca da frequência dos episódios e seus desencadeadores, os dados encontrados na presente pesquisa confirmam o estudo realizado por Magalhães, Lopes, e Marback (2016), no qual $54 \%$ e $44 \%$ dos participantes referiram, respectivamente, dores intensas e medianas a partir da avaliação da EVA; sendo que 47,6\% destes associaram o surgimento da dor aos aspectos emocionais; $40,4 \%$ à alimentação inadequada ou irregular; e $30,9 \%$ à má qualidade do sono.

Sabe-se que fatores relacionados à má qualidade de sono e alimentação podem funcionar como gatilho para a crise de cefaleia (Sahota \& Dexter, 1990; Santana \& Freitas, 2016), esta associada, principalmente, ao jejum prolongado e ao consumo de tipos específicos de alimentos, tais como bebidas alcoólicas e condimentos. Nesse ponto, é importante lembrar que a reeducação alimentar assim como a otimização do sono são estratégias utilizadas na prevenção e no tratamento dessa patologia.
A literatura também tem mostrado que variáveis psíquicas estão relacionadas com a manifestação dos quadros de dor (Tsuji \& Carvalho, 2002). No que tange à depressão e sua manifestação clínica, parte-se da compreensão de que esta atua como uma comorbidade bastante comum nos quadros de cefaleia primária. A esse respeito, Costa et al. (2006) apontaram que o transtorno depressivo maior atinge três vezes mais a população migranosa do que a população geral. Enquanto a presente pesquisa encontrou uma prevalência de $52,5 \%$ de sintomas depressivos de nível leve a grave, Mascella (2011) constatou que $48,3 \%$ dos participantes pesquisados apresentaram nível mínimo de depressão, tendo apresentado sentimentos negativos, tais como raiva, ansiedade, medo e angústia diante da dor. A autora ainda relata que a manifestação dolorosa é marcada por sentimentos de tristeza e inconformismo, bem como estratégias de esquiva/fuga, tais como busca por isolamento. Em seu estudo, Matta e Moreira (2003) encontraram sintomas depressivos em $40 \%$ dos pacientes diagnosticados com cefaleia do tipo tensional episódica. Esses dados confirmam a hipótese inicial de que há uma relação entre depressão e cefaleia.

$\mathrm{Na}$ literatura pesquisada não foram encontrados dados específicos acerca dos aspectos subjetivos relacionados à cefaleia primária. No entanto, alguns estudos sobre dor crônica podem ser utilizados como parâmetro de análise dessa temática. Partindo desse ponto, compreende-se que respostas emocionais de ansiedade e depressão são comuns em quadros clínicos de dor crônica, dentre as quais a cefaleia primária está inclusa, uma vez que a manifestação dolorosa já não possui uma função de alerta no organismo, atuando de forma disfuncional (Flores \& Costa, 2008).

Uma característica do comportamento de dor encontrado em muitos quadros de dor crônica se refere às funções de esquiva de situações vivenciadas como estressoras e/ou aquisição de reforçamento positivo, por meio da proteção e cuidado de terceiros (Flores \& Costa, 2008; Turk \& Akiko, 2002). Tais características não foram percebidas entre os participantes entrevistados na presente pesquisa, visto que a maioria admitiu enfrentar as obrigações cotidianas, mesmo sob influência da dor, além de referir a preferência pelo isolamento social nos momentos de manifestação sintomática da cefaleia. Os seguintes trechos ilustram, respectivamente, ambas as situações:

Sujeito $n^{\circ} 22$ : "(...) eu procuro nem ver ninguém também quando eu tô com dor de cabeça e as pessoas também entendem isso e me deixam sozinha";

Sujeito no 12 "(...) o que eu tenho que fazer, eu cumpro minhas obrigações, mesmo com dor de cabeça. É claro que isso não funciona $100 \%$, mas eu procuro cumprir ao máximo 
minhas obrigações".

Outra característica percebida nesse público é o foco da atenção no problema, o que favorece o desenvolvimento de comportamentos que tornam a vida das pessoas mais influenciada pela dor, sendo exemplos disso o menor engajamento em atividades produtivas/satisfatórias ou de lazer e, consequentemente, a menor percepção de seus benefícios (McCorkle, 1978 como citado em Flores \& Costa, 2008). Esse foi um aspecto visivelmente percebido no discurso dos participantes dessa pesquisa, os quais afirmaram, em sua maioria, possuir uma rotina muito atarefada, mas de modo insatisfatório. Embora trabalhassem, os indivíduos afirmaram que seus rendimentos muitas vezes não atendiam suas expectativas e que não incluíam em sua rotina atividades de lazer.

Outro aspecto percebido nas pessoas acometidas por dores crônicas se refere à antecipação de estados de ansiedade e depressão, bem como sentimentos de desamparo (Rollnik, Karst, Fink, \& Dengler, 2001), aspecto também percebido no discurso dos entrevistados na presente pesquisa, como pode ser observado no seguinte trecho:

Sujeito no 12: "(...) como eu já senti muita dor de cabeça, qualquer sintoma de dor de cabeça que eu sinto já me traz, digamos, um certo pânico".

No que diz respeito às representações simbólicas referidas pelos participantes, que afirmam que a cefaleia "é literalmente uma dor de cabeça", o sujeito relacionou a dor de cabeça aos problemas e dificuldades encontradas por ele no momento da manifestação sintomática, referindo seu sintoma como próprio parâmetro de avaliação negativa; enquanto que, ao dizer que a dor "é um freio, me freia de fazer alguma coisa", remete-se ao caráter incapacitante e limitador que a dor de cabeça possui, principalmente quando se trata da interferência desta em suas atividades diárias e/ou rotina; por fim, quando afirma que a manifestação dolorosa "é uma maldição" e "é uma cruz", explicita o significativo mal-estar sentido sem uma causa aparente.

Em suma, os resultados encontrados na pesquisa convergem com dados disponíveis na literatura (Flores \& Costa, 2004; Mascella, 2011; Santana \& Freitas, 2016; Tsuji \& Carvalho, 2002; Villa, 2015). Os aspectos emocionais, concomitantes com pouca qualidade do sono e a ingestão de determinados tipos de alimentos podem atuar como agentes desencadeadores de crises, podendo ainda haver a manifestação de comorbidades, resultando em prejuízo significativo para as pessoas em seus domínios sociais. Diante do que foi apresentado, salienta-se a necessidade de atenção dos profissionais da área da saúde, no intuito de expandir e aprofundar os estudos acerca dessa temática, tendo em vista uma maior compreensão acerca da cefaleia primária para o desenvolvimento de formas de manejo eficazes, fazendo com que os indivíduos acometidos tenham uma melhor qualidade de vida.

\section{CONSIDERAÇÕES FINAIS}

Conclui-se que os respondentes apresentaram níveis significativos de sintomas depressivos, o que corrobora os dados encontrados na literatura. Notou-se que há um importante comprometimento do aspecto psicológico e subjetivo entre os participantes acometidos, visto que em períodos de crise essas pessoas buscam isolamento social e uso de medicamentos, muitas vezes, sem prescrição médica. Percebeu-se ainda que essas pessoas possuíam sentimentos de tristeza, angústia e impaciência, também apresentando irritabilidade e ansiedade. Tais resultados mostraram que há uma relação entre sintomas depressivos e cefaleia, marcando, assim, a necessidade de maior empenho e atenção dos profissionais de saúde em relação à promoção de saúde psíquica para essas pessoas.

Contudo, é importante salientar as limitações do presente estudo, sobretudo o fato da amostra ter sido composta por conveniência e acessibilidade, o que pode ter limitado a possibilidade de extrapolação para outros indivíduos que apresentavam a cefaleia primária. Além disso, a ausência de uma amostra mais significativa, bem como a falta de estudos com o mesmo objetivo e com a mesma população-alvo para comparação dos dados encontrados podem ser considerados aspectos limitadores.

Finalmente, ressalta-se que o presente estudo pode contribuir com as discussões que já vêm sendo realizadas sobre a temática. Acredita-se que aos profissionais e pesquisadores da área cabe a tarefa de investir no aprofundamento do estudo dessa temática, no intuito de que seja possível compreendê-la de modo mais satisfatório para que as estratégias de enfrentamento e tratamentos propostos às pessoas acometidas pela cefaleia sejam mais eficazes, especialmente do ponto de vista psicológico. Acredita-se na importância da continuação do presente estudo por meio da ampliação do número de participantes e utilização de outros instrumentos de coleta de dados para aprofundamento do tema em questão.

\section{DECLARAÇÃO DE FINANCIAMENTO}

A pesquisa relatada no manuscrito foi financiada pela bolsa de iniciação científica da primeira autora (FAPESB, prot. 4188/2016). 


\section{W INERACÄOEM ET PSICOLOGIA}

\section{DECLARAÇÃO DA CONTRIBUIÇÃO DOS AUTORES}

Administração do projeto: R. F. M.; análise formal dos dados: A. S. S e K. L. S. M; conceitualização: R. F. M.; investigação: A. S. S. e K. L. S. M.; metodologia: R. F. M.; redação - preparação do rascunho original: A. S. S. e K. L. S. M.; redação - revisão e edição: A. S. S., K. L. S. M e R. F. M.; supervisão: R. F. M.; tabulação dos dados: A. S. S. e K. L. S. M.; validação: R. F. M.; visualisação: A. S. S.

\section{DECLARAÇÃO DE CONFLITOS DE INTERESSE}

Os autores declaram que não há conflitos de interesse no manuscrito submetido.

\section{REFERÊNCIAS}

American Psychiatric Association. (2014). DSM-5. American Psychiatric Association. (5 ${ }^{\mathrm{a}}$ Ed.). Porto Alegre: Artmed.

Bardin, L. (1977). Análise de conteúdo. Lisboa: Edições 70.

Beck, A. T; Ward, C. H.; Mendelson, M.; Mock, J., \& Erbaugh, J. (1961). An inventory for measuring depression. Archives of general psychiatry, 4(6), p. 561-571.

Bigal, Bordini \& Speciali (1999). Tratamento da cefaléia em uma unidade de emergência da cidade de Ribeirão Preto. Arquivos de Neuro-Psiquiatria, 57(3B), 813-819. DOI: 10.1590/S0004-282X1999000500013

Brasil. (2012). Resolução 466/2012. Diretrizes e normas regulamentadoras de pesquisas envolvendo seres humanos. Ministério da Saúde/Conselho Nacional de Saúde. Brasília.

Breslau, N.; Davis, G.; Schultz, L., \& Peterson, E. (1994). Migraine and major depression: a longitudinal study. Headache, 34(7), 387-393. DOI: 10.1111/j.15264610.1994.hed3407387

Costa, E.; Ybarra, M.; Corrêa, H., \& Teixeira, A. (2006). Enxaqueca e depressão: comorbidade ou espectro. RBM Revista Brasileira de Medicina, 63(8), 392-5.

Feldman, A. (1995). Cefaleias primárias. Porto alegre: Artes Médicas.

Flores, A., \& Costa Junior, A. (2004). O manejo psicológico da dor de cabeça tensional. Psicologia, Ciência e Profissão, 24(3), 24-33. DOI: 10.1590/S1414-98932004000300004.

Flores, A., \& Costa Júnior, A. (2008). Modelo biopsicossocial e formulação comportamental: compreendendo a cefaléia do tipo tensional. Psicologia em estudo, 13(1), 143-151. DOI: $10.1590 / S 1413-73722008000100017$

Freud, S. (1893). Sobre os mecanismos psíquicos dos fenômenos histéricos: uma conferência. (Vol. 3). Rio de Janeiro: Imago.
Krymchantowski, A. (2008). Condutas em cefaleia: avaliação e tratamento. São Paulo: Lippincott Williams \& Wilkins.

Lima M. \& Trad L. (2007). A dor crônica sob o olhar médico: modelo biomédico e prática clínica. Cadernos de Saúde Pública, 23(11), 2672-80.

Magalhães, L.; Lopes, T., \& Marback, R. (2016). Interferências na rotina e na qualidade de vida provocadas pela cefaleia primária. Seminário Estudantil de Produção Acadêmica, 15, 285-296.

Mascella, V. (2011). Stress, sintomas de ansiedade e depressão na migrânea e cefaléia tensional (Dissertação de mestrado). Pontifícia Universidade Católica de Campinas, Campinas.

Matta, A., \& Moreira Filho, P. (2003). Sintomas depressivos e ansiedade em pacientes com cefaléia do tipo tensional crônica e episódica. Arquivos de Neuro-Psiquiatria, 61(4), 991-994. DOI: 10.1590/S0004-282X2003000600019

Morais, M., \& Benseñor, I. (2009). Cefaleias primárias. Revista Brasileira de Medicina, 66(6), 138-147.

Nobre, M. (2006). Cefaleias em salvas. São Paulo: Lemos Editorial.

Organização Mundial da Saúde (OMS). (2001). Relatório sobre a saúde no mundo. Saúde Mental: nova concepção, nova esperança. Genebra.

Petersen, C., \& Nunes, M. (2002). Cefaleia tensional crônica e psicopatologia. Psic: revista da Vetor Editora, 3(2), 30-43.

Pinto, M., Wagner, H., Klafke, A., Ramos, A., Stein, A., \& Castro Filho, E. (2007). Diagnóstico e Tratamento das cefaléias em adultos na Atenção Primária à Saúde. Sociedade Brasileira de Medicina de Família e Comunidade, 17.

Rollnik, J., Karst, M., Fink, M., \& Dengler, R. (2001). Coping strategies in episodic and chronic tension-type headache. Headache, 41(4), 297-302. DOI: 10.1046/j.15264610.2001.111006297.x

Rubbo, A. B. (2010). Escala Visual Analógica na avaliação da intensidade da dor pós-operatória de cirurgia bariátrica independente do uso de analgésicos (Tese de doutorado). Faculdade de Ciências Médicas da Santa Casa de São Paulo, São Paulo.

Sahota, R., \& Dexter, J. (1990). Sleep and headache syndromes: a clinical review. Headache: The Journal of Head and Face Pain, 30(2), 80-84. DOI: 10.1111/j.15264610.1990.hed3002080.x

Santana, L., \& Freitas, B. (2016). Influência dietética e nutricional na migrânea. Journal of Health Sciences, 18(1), 64-73. DOI: 10.17921/2447-8938.2016v18n1p64-73

Speciali, J. (1997). Classificação das cefaléias. Medicina, Ribeirão Preto, 30, 421-427. DOI: 10.11606/issn.21767262.v30i4p421-427 


\section{N"INTERACÃO EM ET. PSICOLOGIA}

Subcomitê de Classificação das Cefaleias da Sociedade Internacional das Cefaleias (2006). Classificação Internacional das Cefaléias (2a Ed.) São Paulo: Alaúde Editorial Ltda.

Turk, D., \& Akiko, O. (2002). Psychological factors in chronic pain: Evolution and revolution. Journal of Consulting and Clinical Psychology, 70(3), 678-690. DOI: 10.1037/0022006X.70.3.678

Tsuji, S., \& Carvalho, D. (2002). Aspectos Psíquicos das Cefaléias Primárias. Revista de Neurociências, 10(3), 129136.
Villa, T. (2015). A automedicação agrava as dores de cabeça crônicas. Retirado de: http://www.minhavida.com.br/ saude/materias/14593-a-automedicacao-agrava-asdores-decabeca-cronicas

Zukerman, E., Guendler, V., Mercante, J., \& Peres, M. (2004). Cefaléia e qualidade de vida. Einstein, 2(1), 73-75.

Data de submissão: 19/04/2017 Primeira decisão editorial: 13/07/2018 Aceite: 15/02/2019 